\title{
Interacting with Next-Generation Smart Patient-Centric Healthcare Systems
}

\author{
Juliana Bowles, Juan Mendoza-Santana, Thais Webber \\ $\{j k f b, j j m 20, t c w d s\} @ s t-a n d r e w s . a c . u k$ \\ School of Computer Science, University of St Andrews \\ St Andrews, UK
}

\begin{abstract}
There is a recognised move towards more personalised health with citizens at the centre of healthcare provision. In particular, there is an emphasis on the right of citizens to decide who, why and when, should have access to their medical records. The EU project SERUMS is developing a tool-chain for the secure access of distributed medical information preserving the privacy levels imposed by GDPR, national and/or organisational regulations. We propose a user-centred approach to demonstrate how technologies can converge to enable doctors and patients to interact with integrated healthcare records. In addition, it will allow us to evaluate and evolve our tool-chain.
\end{abstract}

\section{KEYWORDS}

Smart healthcare, Patient-Centric, User interaction

\section{ACM Reference Format:}

Juliana Bowles, Juan Mendoza-Santana, Thais Webber. 2020. Interacting with Next-Generation Smart Patient-Centric Healthcare Systems. In Adjunct Proceedings of the 28th ACM Conference on User Modeling, Adaptation and Personalization (UMAP '20 Adjunct), fuly 14-17, 2020, Genoa, Italy. ACM, New York, NY, USA, 2 pages. https://doi.org/10.1145/3386392.3399561

\section{INTRODUCTION}

The EU project SERUMS ${ }^{1}$ brings together several technologies such as novel authentication mechanisms [1], privacy-preserving approaches [3], and emerging blockchain concepts and features [4] to create the SERUMS portal for secure health data provision and data analytics. It is a multi-layered and decentralised patient-centric approach [2] for transnational data exchanges. SERUMS integrates and aggregates patient records in a centralised data lake as Smart Patient Health Records (SPHR). SPHR is a universal format proposed by SERUMS to represent the metadata that describe the medical records present in distributed sources (hospitals and out-of-hospital environments) across Europe. It is a challenge to preserve the security and privacy of the patient data at all times, whilst complying to different national and international legislations (such as GDPR) that regulate ownership and sharing of that data.

\footnotetext{
${ }^{1}$ For more information see www.serums-h2020.org.

Permission to make digital or hard copies of all or part of this work for personal or classroom use is granted without fee provided that copies are not made or distributed for profit or commercial advantage and that copies bear this notice and the full citation on the first page. Copyrights for components of this work owned by others than ACM must be honored. Abstracting with credit is permitted. To copy otherwise, or republish, to post on servers or to redistribute to lists, requires prior specific permission and/or a fee. Request permissions from permissions@acm.org.

UMAP '20 Adjunct, fuly 14-17, 2020, Genoa, Italy

(c) 2020 Association for Computing Machinery.

ACM ISBN 978-1-4503-7950-2/20/07 . \$ $\$ 15.00$

https://doi.org/10.1145/3386392.3399561
}

\section{SERUMS SYSTEM DESIGN}

SERUMS integrates a variety of technologies into a coherent Smart Health Centre System (SHCS) with a centralised front-end to allow: (i) patients to have more control over their personal medical data and (ii) professionals to make better-informed decisions based on a more holistic view of patient records.

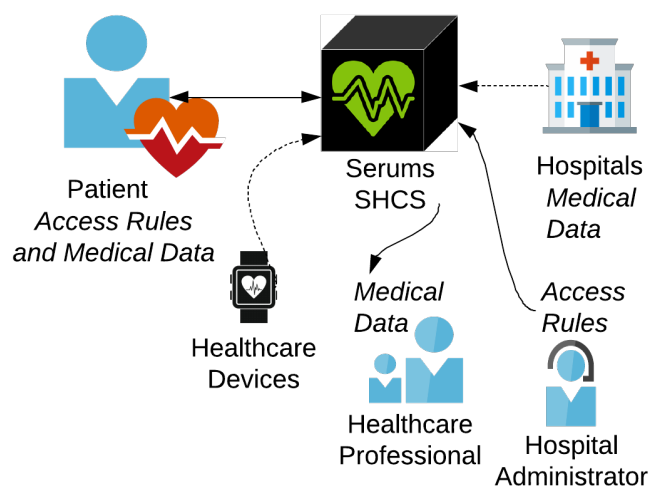

Figure 1: SERUMS' user context and relevant interactions

Figure 1 illustrates the SERUMS' user perspective, where patients are empowered to define individual access rules for (parts of) their medical records. Doctors are allowed to access integrated patient records in a meaningful manner. Several other healthcare professionals can be more confident in taking clinical or operational decisions based on real-time data, depending on their role in the healthcare organisation. Further information systems (e.g. health devices, trackers, hospital information systems, etc.) can also be integrated with customised APIs to securely exchange medical data.

SERUMS has a especial concern regarding the interaction of end users with the system, especially the patients, when performing their tasks. For this reason, it follows a user-centred design approach, which is supported by three different use cases (UCs) ${ }^{2}$. Diverse hospitals and medical centres (dispersed in different countries) support our synergistic design process. They allow the engagement of several researchers and potential users to construct functional scenarios for diverse interaction situations. For a user-centred design to be effective when proposing such healthcare system, it is essential to find out as much information as possible about the users' needs, both from the perspective of the patients and the healthcare organisations.

\footnotetext{
${ }^{2}$ Current UCs include Zuyderland Medisch Centrum (ZMC) in the Netherlands, Hospital Clinic de Barcelona (HCB) in Catalonia, and the Edinburgh Cancer Data (ECD) in Scotland.
} 
The high-level user requirements aim to facilitate user navigation and medical data retrieval, and consequently should make all system functionalities intuitive, from patient search function to further options such as visualisation of patient records. In order to enable these functionalities in the front-end, the back-end must coordinate the blockchain authorisation mechanism with the integrated T-PO-L-E (Time, Person, Object, Location, Event) data vault, provided by underlying data lake implementation. Doctors should be able to access information quickly and easily, thus we favour a minimalist user interface that provides direct access to all required information.

The user interface must show medical information in a structured, organised, and navigable manner. Following the SERUMS data vault model to retrieve the SPHR, the information is structured as a list of events, with key data provided by the T-P-O-L-E concept.

Patients have control over their personal clinical information, so they decide how their information will be available. This makes it necessary to provide the means to customise access rules in a intuitive manner. This requirement is fulfilled by a formal language for the permission rules, which is hidden underneath an interface that enables patients and other authorised users to manipulate these rules in natural language (Figure 2). The verification of access rules follows a logic-based approach enabling conflict-free layers of authorisation, with priority levels guided by government laws, organisation policies and individual rules.

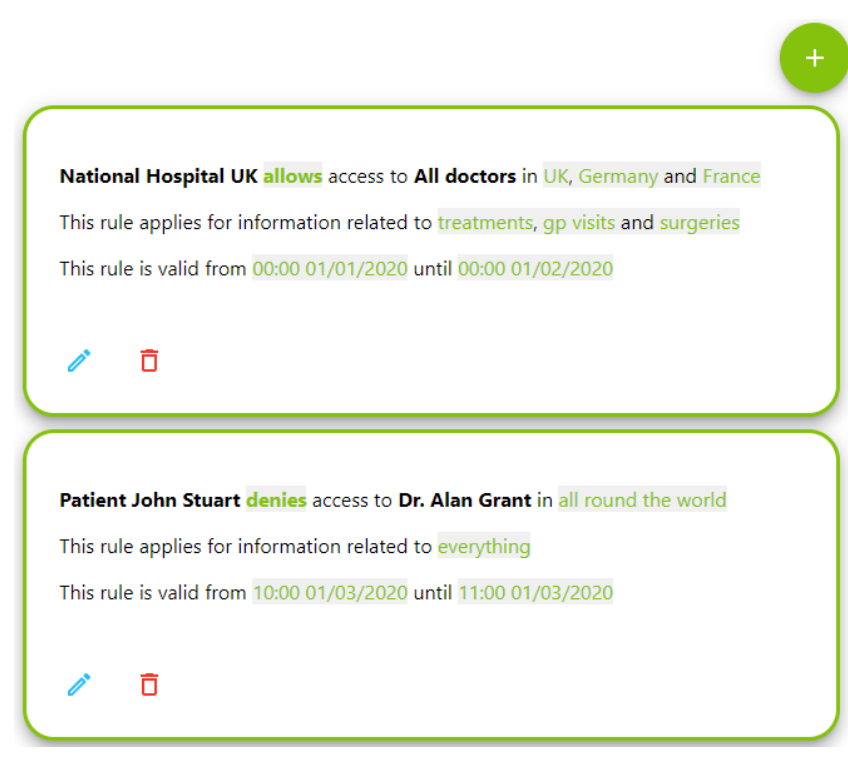

Figure 2: Access Rules definition interface

Envisioning global healthcare systems demands a high-level system interaction, and we provide a public API (Figure 3) to make SERUMS technologies accessible to other platforms.

\section{CONCLUSION}

The SHCS front-end and backend features are built to demonstrate the effectiveness of the integration of SERUMS technologies, and the adequate response to authorised users. At this stage of the project we focus on exploring alternatives for user interfaces centred on the

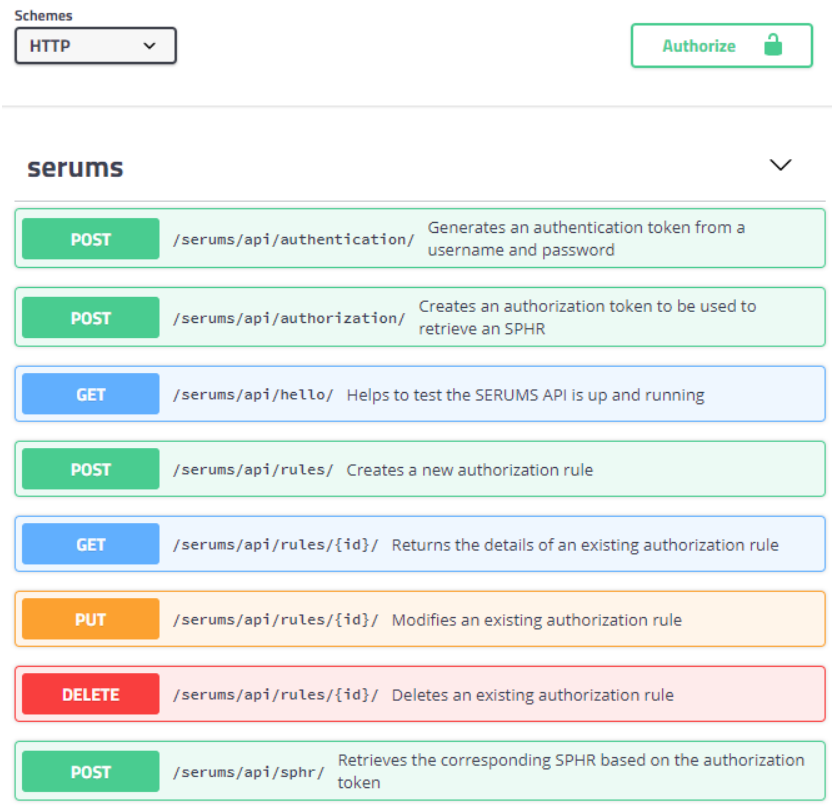

Figure 3: SERUMS Technologies API

needs of doctors and patients, and how to encode rules that capture that. In our approach, patients can make decisions about who they are willing to share their medical data with, and for how long. Doctors can explore the benefits of accessing integrated healthcare records from distributed sources. At the same time, the system preserves security and privacy-preserving properties encoded in the authentication, authorisation and data components of the SERUMS tool-chain [2].

In the near future we will be placing our system in a real context by engaging our hospital partners in the design and implementation of experiments regarding user experience. In this way, we will be able to evaluate aspects of usability and integration.

\section{ACKNOWLEDGMENTS}

This research is funded by the EU H2020 project SERUMS: Securing Medical Data in Smart Patient-Centric Healthcare Systems (grant code 826278 ).

\section{REFERENCES}

[1] M. Belk, C. Fidas, and A. Pitsillides. 2019. FlexPass: Symbiosis of Seamless User Authentication Schemes in IoT. In Extended Abstracts of the 2019 CHI Conf. on Human Factors in Computing Systems. ACM, New York, NY, USA, Article LBW2318, 6 pages.

[2] V. Janic, J.K.F. Bowles, A.F. Vermeulen, and et al. 2019. The Serums tool-chain: Ensuring Security and Privacy of Medical Data in Smart Patient-Centric Healthcare Systems. In 2019 IEEE International Conference on Big Data (Big Data). IEEE, Los Angeles, CA, USA, 2726-2735.

[3] M. Kumar, M. Rossbory, B. A. Moser, and B. Freudenthaler. 2019. Deriving an Optimal Noise Adding Mechanism for Privacy-Preserving Machine Learning. In Database and Expert Systems Applications (DEXA 2019) (Communications in Computer and Information Science), G. Anderst-Kotsis and et al. (Eds.), Vol. 1062. Springer, Cham, 108-118.

[4] A. Singh, R. M. Parizi, Q. Zhang, K.-K. R. Choo, and A. Dehghantanha. 2020. Blockchain smart contracts formalization: Approaches and challenges to address vulnerabilities. Computers \& Security 88 (2020), 101654. 